\title{
Nota Científica \\ Qualidade morfológica de mudas de Prunus sellowii submetidas a diferentes substratos
}

\author{
Adriana Falcão Dutra ${ }^{*}$, Maristela Machado Araujo1 ${ }^{1}$, Anna Paula Lora Zimmermann ${ }^{1}$, Daniele Guarienti Rorato ${ }^{1}$, \\ Suelen Carpenedo Aimi ${ }^{1}$
}

${ }^{1}$ Universidade Federal de Santa Maria, Campus Universitário, Camobi, CEP 97105-900, Santa Maria, RS, Brasil

*Autor correspondente:
adriflorestal@gmail.com

Termos para indexação:

Espécie nativa

Regeneração da floresta

Produção de plantas

Index terms:

Native species

Forest rehabilitation

Plant production

Histórico do artigo:

Recebido em 08/09/2015

Aprovado em 17/10/2017

Publicado em 29/12/2017

doi: 10.4336/2017.pfb.37.92.1030
Resumo - O objetivo desse trabalho foi avaliar diferentes proporções de casca de arroz carbonizada em substrato comercial, no crescimento de mudas de Prunus sellowii em viveiro. Os tratamentos foram compostos de: T1 - 100\% substrato comercial (SC); T2 - 90\% SC e 10\% casca de arroz carbonizada (CAC); T3-80\% SC e 20\% CAC; T4 -70\% SC e 30\% CAC; $\mathrm{T} 5$ - 60\% SC e 40\% CAC; T6 - 50\% SC e 50\% CAC, em delineamento experimental inteiramente casualizado, com quatro repetições. Recomenda-se a adição de até $30 \%$ de CAC, sem prejuízos ao crescimento dessa espécie em viveiro.

\section{Morphological quality of Prunus sellowii seedlings under different substrates}

\begin{abstract}
This study aimed to evaluate different proportions of carbonized rice husk on commercial substrate, in the growth of Prunus sellowii seedlings in nursery. The treatments were composed of: T1-100\% commercial substrate (SC); T2 - 90\% SC and $10 \%$ carbonized rice husk (CRH); T3 - 80\% SC and 20\% CRH; T4 - 70\% $\mathrm{SC}$ and $30 \% \mathrm{CRH}$; T5 - 60\% SC and 40\% CRH; T6 -50\% SC and 50\% CRH, in a completely randomized design, with four replications. The addition of up to $30 \%, \mathrm{CRH}$ is recommended, without harm to $P$. sellowii growth in nursery.
\end{abstract}

\section{Introdução}

A redução da cobertura florestal oriunda de constantes supressões, para dar origem a novas áreas agrícolas e urbanas, acarreta paisagens biologicamente alteradas (Bertacchi et al., 2016), demandando intervenções no restabelecimento dessas áreas. Para a recuperação ambiental de áreas alteradas ou degradadas, pesquisas indicam como uma das alternativas o plantio de mudas de espécies arbóreas nativas, que pode ser viabilizado pela combinação de espécies de diferentes grupos ecológicos e funcionais (Campoe et al., 2014; Ferez et al., 2015). Entretanto, para que essa técnica seja efetiva, ressalta-se a importância de pesquisas considerando a silvicultura de espécies nativas com potencial ecológico (Rorato et al., 2017), desde as etapas de produção das mudas em viveiro até o plantio e monitoramento em campo.

Uma das espécies interessantes para essa finalidade é Prunus sellowii Koehne (pessegueiro-do-mato) da 
família Rosaceae, cujo porte arbóreo pode atingir até 25 $\mathrm{m}$ de altura e $80 \mathrm{~cm}$ de diâmetro. A espécie floresce de agosto a março e de dezembro a fevereiro, frutificando, abundantemente, de janeiro a outubro e de junho a agosto (Backes \& Irgang, 2009). Segundo esses autores, a polinização é realizada por abelhas e a dispersão dos frutos por diversas aves, o que favorece a polinização. Tal circunstância, aliada ao rápido crescimento da espécie, confirma a importância ecológica de $P$. sellowii para recuperação ambiental.

O sucesso na implantação de plantios em campo está fortemente relacionado com a qualidade das mudas utilizadas (Ferrari et al., 2016). Conforme Gonçalves et al. (2012) e Dutra et al. (2016), estudos envolvendo fatores como substrato, adubação e irrigação na produção de mudas de espécies nativas em viveiro, possibilitam a qualificação do material disponibilizado aos plantios, viabilizando as atividades silviculturais.

Dentre esses insumos, a escolha adequada do substrato é um dos fatores que interfere na qualidade de mudas produzidas em recipientes. Assim, os substratos bem como as suas proporções são continuamente investigados (Regan, 2014). Isso é atribuído, principalmente, às características físicas, químicas e biológicas dos materiais utilizados, que são influenciadas pela origem, forma de produção e proporções dos componentes (Melo et al., 2014). A casca de arroz é um material amplamente disponível em regiões com economia baseada em orizicultura. Esse, quando carbonizado, pode ser utilizado como componente de substrato, reduzindo o custo de produção das mudas.

O presente trabalho teve como objetivo avaliar o efeito de diferentes proporções de casca de arroz carbonizada adicionadas a substrato comercial no crescimento de mudas de $P$. sellowii em viveiro.

O experimento foi conduzido de novembro de 2008 a junho de 2009, no Viveiro Florestal da Universidade Federal de Santa Maria(UFSM), localizado no município de Santa Maria, RS $\left(29^{\circ} 43^{\prime}\right.$ 'S e $\left.53^{\circ} 43^{\prime} \mathrm{W}\right)$, sob altitude de $90 \mathrm{~m}$ a $95 \mathrm{~m}$. O clima da região é subtropical úmido, tipo $\mathrm{Cfa}$, caracterizado por apresentar temperatura média do mês mais frio entre $-3{ }^{\circ} \mathrm{C}$ e $18{ }^{\circ} \mathrm{C}$, e do mês mais quente superior a $22{ }^{\circ} \mathrm{C}$, com precipitação média anual de $1.769 \mathrm{~mm}$ e chuvas bem distribuídas ao longo do ano (Alvares et al., 2013).

As sementes de P. sellowii foram fornecidas pelo subprograma Bolsa de Sementes (convênio Associação dos Fumicultores do Brasil/UFSM), coletadas de cinco árvores matrizes com distância mínima entre elas de $100 \mathrm{~m}$, no município de Santa Cruz do Sul, RS, Brasil. Para a produção das mudas foram utilizados tubetes cônicos de polipropileno com capacidade de $280 \mathrm{~cm}^{3}$, acondicionados em bandejas suspensas a $16 \mathrm{~cm}$ da superfície do solo. O substrato comercial (SC) utilizado foi composto de casca de pínus bioestabilizada e vermiculita, sendo adicionadas diferentes proporções de casca de arroz carbonizada (CAC). Na adubação de base (adubo mineral de pronta liberação), foi utilizado $\mathrm{NPK}$, na formulação $35 \mathrm{~g} \mathrm{NH}_{4} \mathrm{NO}_{3}, 400 \mathrm{~g} \mathrm{de} \mathrm{P}_{2} \mathrm{O}_{5} \mathrm{e}$ $20 \mathrm{~g} \mathrm{KCl}$, a cada $100 \mathrm{~L}$ de substrato.

A semeadura foi efetuada diretamente nos tubetes, com três sementes por recipiente. Quando as mudas apresentavam cerca de $5 \mathrm{~cm}$ de altura e dois pares de folhas, foi realizado o raleio, deixando-se apenas a mais vigorosa. As avaliações dos atributos morfológicos foram efetuadas a cada 30 dias, medindo-se altura $(\mathrm{H})$, com auxílio de régua $(\mathrm{cm})$ e diâmetro do coleto (DC), com paquímetro digital de precisão milimétrica.

O experimento foi encerrado aos 150 dias após a emergência (DAE), sendo avaliados os seguintes atributos: massa seca aérea (MSA) e massa seca das raízes (MSR). Para isso, a parte aérea e o sistema radicular foram separados, sendo as raízes lavadas sobre peneira (malha $1 \mathrm{~mm}$ ) em água corrente, a fim de retirar o substrato aderido. Após esse processo, o material foi acondicionado em sacos de papel Kraft e seco em estufa, com circulação forçada de ar, a temperatura de $70{ }^{\circ} \mathrm{C}$, até peso constante, seguido da pesagem em balança analítica de precisão.

A partir desses dados, foi possível avaliar a relação massa seca aérea e massa seca das raízes (MSA/MSR), massa seca total (MST) e o índice de qualidade de Dickson (IQD), conforme equação1(Dickson et al., 1960):

$\mathrm{IQD}=\frac{\mathrm{MST}(\mathrm{g})}{\left[\frac{\mathrm{H}(\mathrm{cm})}{\mathrm{DC}(\mathrm{mm})}\right]+\left[\frac{\mathrm{MSA}(\mathrm{g})}{\mathrm{MRS}(\mathrm{g})}\right]}$

Sendo: $\mathrm{IQD}=$ índice de qualidade de Dickson; $\mathrm{MST}=$ massa seca total; $\mathrm{H}=$ altura; $\mathrm{DC}=$ diâmetro do coleto; $\mathrm{MSA}=$ massa seca da parte aérea; MSR = massa seca das raízes.

O delineamento experimental utilizado foi inteiramente casualizado, com seis formulações de substratos (T1 $100 \%$ SC; T2 - 90\% SC e 10\% CAC; T3 - 80\% SC e $20 \%$ CAC; T4 - 70\% SC e 30\% CAC; T5 - 60\% SC e $40 \%$ CAC; $\mathrm{T} 6-50 \%$ SC e $50 \%$ CAC), com quatro 
repetições cada, cujas variáveis $\mathrm{H}$ e DC foram avaliadas no tempo (30, 60, 90, 120 e $150 \mathrm{DAE})$. Cada parcela (repetição) foi composta por 24 mudas, sendo avaliados os oito indivíduos centrais para as variáveis H e DC. Os demais atributos (MSA, MSR, MST, MSA/MSR e IQD) foram avaliados aos $150 \mathrm{DAE}$, utilizando-se quatro mudas por repetição.

Inicialmente, os dados foram submetidos às pressuposições de normalidade de resíduos e homogeneidade de variâncias, pelos testes de ShapiroWilk e Bartlett, respectivamente. Posteriormente, foram submetidos à análise de variância e quando detectada significância, as varáveis H e DC foram analisadas por regressão, enquanto os demais atributos foram comparados pelo teste de Tukey a $5 \%$ de probabilidade de erro, com o programa SISVAR v. 5.3 (Ferreira, 2014).

As diferentes proporções de casca de arroz carbonizada adicionadas ao substrato apresentaram efeito sobre a altura $(\mathrm{H})$ das mudas de $P$. sellowii. Aos $30 \mathrm{DAE}$, os tratamentos T2 e T3 apresentaram as maiores médias de H (14,2 e 14,1 cm, respectivamente). Entretanto, o

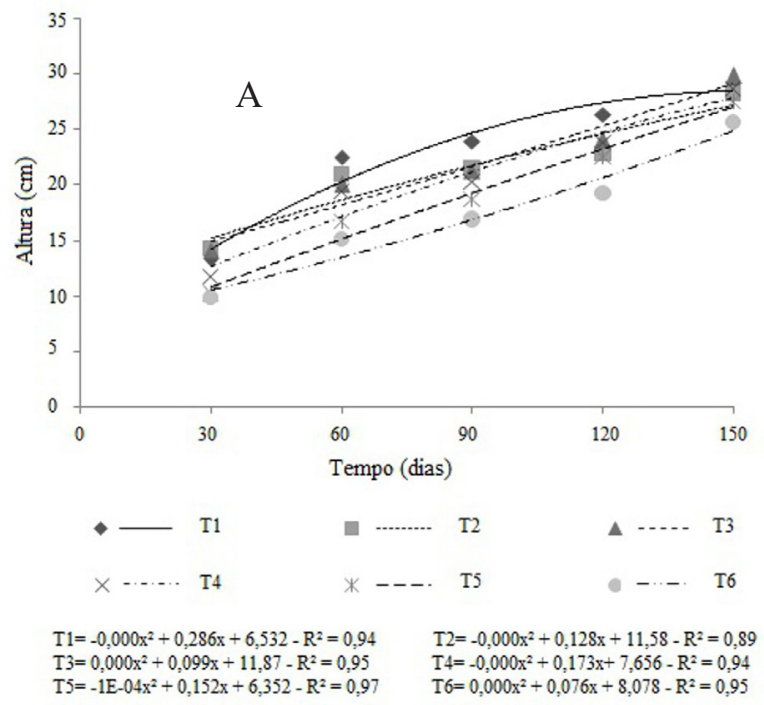

T1 demonstrou crescimento expressivo em $\mathrm{H}$ até os 120 DAE, equiparando-se a T3 aos 150 DAE (Figura 1a). Esse comportamento foi semelhante para o diâmetro do coleto (DC) (Figura 1b), que apresentou médias superiores também em $\mathrm{T} 1$ e $\mathrm{T} 2$, quando as mudas atingiram aproximadamente $3,0 \mathrm{~mm}$.

Corroborando com esse resultado, mudas de espécies florestais nativas como Parapiptadenia rigida e Luehea divaricata, que também apresentam rápido crescimento, podem ser produzidas com até $20 \%$ de CAC misturada ao substrato (Dutra et al., 2016; Mezzomo et al., 2017). Por outro lado, Cabralea canjerana, que tem crescimento lento a moderado, pode ser produzida em substrato composto por $40 \%$ de CAC, sem prejuízo ao crescimento das mudas em campo (Gasparin et al., 2014). Apesar de as espécies arbóreas apresentarem demandas diferenciadas quanto ao substrato, a adição de um componente alternativo ao meio de cultivo em viveiros florestais, em diferentes proporções, possibilita a redução dos custos de produção e a garantia da obtenção de mudas com a qualidade necessária.

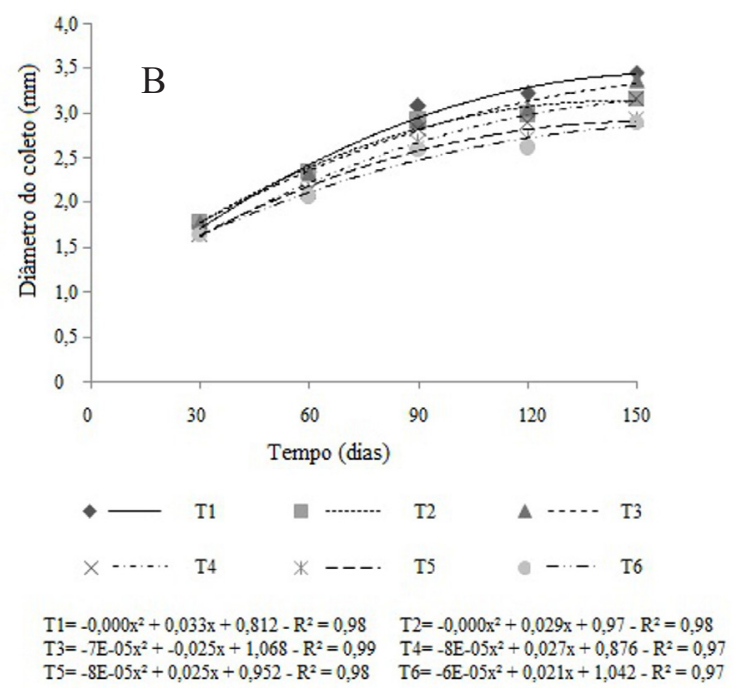

Figura 1. Altura (A) e diâmetro do coleto (B) de mudas de Prunus sellowii aos 30, 60, 90, 120 e 150 dias após a emergência (DAE), em função dos diferentes substratos (T1 - 100\% substrato comercial (SC); T2 - 90\% SC e $10 \%$ casca de arroz carbonizada (CAC); T3 - $80 \%$ SC e $20 \%$ CAC; T4 - $70 \%$ SC e $30 \%$ CAC; T5 - $60 \%$ SC e $40 \%$ CAC; e T6 - 50\% SC e 50\% CAC), com as respectivas equações e coeficientes de determinação $\left(\mathrm{R}^{2}\right)$.

Adicionalmente, é possível evidenciar que proporções de $40 \%$ e $50 \%$ de CAC misturadas ao SC, proporcionaram menor crescimento em $\mathrm{H}$ e DC de mudas de $P$. sellowii, com $27,5 \mathrm{~cm}$ e $25,7 \mathrm{~cm}$ e $3,0 \mathrm{~mm}$ e $2,9 \mathrm{~mm}$, respectivamente, aos 150 DAE (Figuras 1a e 1b). O menor incremento é resultante da maior lixiviação dos nutrientes proporcionada pela $\mathrm{CAC}$, principalmente, devido à adubação de pronta liberação utilizada nesse experimento. Esse componente, que apresenta baixa capacidade de retenção de água, boa aeração, baixa densidade e pH em torno da neutralidade (Kämpf, 2005; Soares et al., 2012), demonstrou que o acúmulo de biomassa de $P$. sellowii é inversamente proporcional ao acréscimo de CAC no substrato. 
Contudo, cabe ressaltar que a partir dos 60 DAE observou-se H superior a $15 \mathrm{~cm}$ e DC de, aproximadamente, 2,5 $\mathrm{mm}$ para a maioria dos substratos (Figuras $1 \mathrm{a} \mathrm{e} 1 \mathrm{~b}$ ), demonstrando rápido crescimento da espécie em viveiro, o que reforça seu potencial para uso em recuperação de áreas degradadas (Backes \& Irgang, 2009).

Com base nos valores de massa seca (Tabela 1), constatou-se semelhança entre os tratamentos com até
$30 \%$ de CAC. A MSA relaciona-se com a produtividade, pois as folhas constituem uma das principais fontes de fotoassimilados (açúcares, aminoácidos, hormônios, entre outros) e nutrientes necessários para o suprimento das necessidades da planta, principalmente, no primeiro mês de plantio, quando realocam as reservas da copa para a síntese de raízes (Bellote \& Silva, 2000).

Tabela 1. Massa seca aérea (MSA), massa seca de raízes (MSR), massa seca total (MST), relação massa seca aérea/ massa seca de raízes (MSA/MSR) e índice de qualidade de Dickson (IQD), de mudas de Prunus sellowii, aos 150 dias após a emergência.

\begin{tabular}{lccccc}
\hline \multicolumn{1}{c}{ Tratamento } & MSA (g) & MSR (g) & MST (g) & MSA/MSR (g) & IQD \\
\hline T1 - 100\% SC & $5,55 \mathrm{a}$ & $1,92 \mathrm{a}$ & $7,51 \mathrm{a}$ & $2,86 \mathrm{a}$ & $0,65 \mathrm{a}$ \\
$\mathrm{T} 2-$ 90\% SC e 10\% CAC & $3,90 \mathrm{ab}$ & $1,61 \mathrm{a}$ & $5,51 \mathrm{ab}$ & $2,39 \mathrm{ab}$ & $0,48 \mathrm{c}$ \\
$\mathrm{T} 3-80 \%$ SC e 20\% CAC & $3,47 \mathrm{ab}$ & $1,73 \mathrm{a}$ & $5,20 \mathrm{ab}$ & $2,00 \mathrm{ab}$ & $0,51 \mathrm{~b}$ \\
T4 - 70\% SC e 30\% CAC & $3,93 \mathrm{ab}$ & $1,69 \mathrm{a}$ & $5,62 \mathrm{ab}$ & $2,33 \mathrm{ab}$ & $0,48 \mathrm{c}$ \\
T5 - 60\% SC e 40\% CAC & $2,52 \mathrm{~b}$ & $1,35 \mathrm{a}$ & $3,88 \mathrm{~b}$ & $1,92 \mathrm{~b}$ & $0,34 \mathrm{~d}$ \\
T6 - 50\% SC e 50\% CAC & $2,93 \mathrm{~b}$ & $1,31 \mathrm{a}$ & $4,23 \mathrm{~b}$ & $2,29 \mathrm{ab}$ & $0,33 \mathrm{e}$ \\
\hline
\end{tabular}

$\mathrm{SC}=$ substrato comercial a base de casca de pínus bioestabilizada e vermiculita; $\mathrm{CAC}=$ casca de arroz carbonizada. Médias seguidas da

mesma letra na coluna não diferem entre si pelo teste de Tukey a $5 \%$ de probabilidade de erro.

Por outro lado, a MSR não apresentou diferença, possivelmente devido às condições fornecidas pelo substrato e pelo manejo aplicado, que favoreceu a absorção hídrica e nutricional em todos os tratamentos. A quantificação da biomassa radicular é importante, tendo em vista que mudas que apresentam maior síntese radicular estão menos vulneráveis ao estresse ambiental após o plantio, por possuírem maior facilidade de sustentação, além de maior superfície e eficiência para absorção de água e nutrientes (Freitas et al., 2005). Entretanto, nesse estudo, a MSR, não discriminou os tratamentos, o que ressalta a necessidade da análise conjunta de todos os atributos para definir protocolos de produção de mudas em viveiro.

Além disso, apesar da maior relação MSA/MSR ter sido observada em $100 \%$ SC (T1), essa também não diferiu de T2, T3, T4 e T6, demonstrando proporcionalidade na alocação do carbono entre esses tratamentos. Conforme Cruz et al. (2010), a produção de massa seca das plantas está relacionada com um melhor vigor e capacidade fotossintética, sendo desejável que encontrem no seu máximo.

Destaca-se que o único atributo que permitiu distinguir efetivamente um dos substratos foi o IQD, que demonstrou melhor resultado para T1 $(0,65)$ (Tabela 1).
O IQD é considerado um eficiente indicador da qualidade das mudas, pois no seu cálculo são consideradas a altura e o diâmetro do coleto e o equilíbrio da distribuição da biomassa na muda (Fonseca et al., 2002). Conforme Davide et al. (2015), maiores valores indicam maior qualidade das mudas para expedição a campo. Nesse sentido, valores superiores a 0,20 são adequados para indicar a qualidade superior de mudas (Gomes \& Paiva, 2011), o que foi observado em todos os tratamentos (Tabela 1).

Salienta-se que o IQD, por considerar diversos atributos, muitas vezes impossibilita comparações, quando utilizam-se diferentes insumos e manejo. Nesse sentido, ao analisar o estudo de Knapik \& Angelo (2007) com a mesma espécie e diferentes adubações de base, foi observado IQD $=0,13$. A H e o DC, apesar de similares às constatadas nesse estudo, apresentaram massa seca aérea e de raízes expressivamente menores, reduzindo consequentemente os valores encontrados no IQD.

Em estudos semelhantes, Saidelles et al. (2009) observaram que, de modo geral, ocorreu maior crescimento à medida que se aumentou a adição de CAC no solo com mudas de Enterolobium contortisiliquum e Apuleia leiocarpa. Nessa situação, o crescimento máximo foi observado na mistura de $50 \%$ de CAC, demonstrando que a proporção adicionada de componente alternativo 
(CAC) pode ser variável, dependendo da espécie.

Assim, aos $150 \mathrm{DAE}$, mudas produzidas com a adição de até $30 \%$ de CAC ao substrato apresentaram qualidade superior, com H e DC superiores a $20 \mathrm{~cm}$ e $3 \mathrm{~mm}$, respectivamente. Em termos práticos, o estabelecimento inicial das mudas em campo será favorecido, aumentando as taxas de sobrevivência, e consequentemente os custos com replantio dos indivíduos será reduzido. Cabe ressaltar que a adubação de cobertura na produção de mudas de $P$. sellowii, principalmente a partir dos 60 DAE, poderá ser uma estratégia para o incremento em H, DC, massa seca e IQD das mudas produzidas com até $30 \%$ de CAC.

\section{Conclusão}

Na produção de mudas de Prunus sellowii, visando à redução nos custos de produção, recomenda-se a adição de até $30 \%$ de casca de arroz carbonizada, sem prejuízos ao crescimento dessa espécie em viveiro.

\section{Referências}

Alvares, C. A. et al. Köppen's climate classification map for Brazil. Meteorologische Zeitschrift, v. 22, n. 6, p. 711-728, 2013. DOI: 10.1127/0941-2948/2013/0507.

Backes, P. \& Irgang, B. Árvores do Sul: guia de identificação e interesse ecológico. 2. ed. Porto Alegre: Paisagem do Sul, 2009. $322 \mathrm{p}$.

Bellote, A. F. J. \& Silva, H. D. Técnicas de amostragem e avaliações nutricionais em plantios de Eucalyptus spp. In: Gonçalves, J. L. M. \& Benedetti, V. (Ed.). Nutrição e fertilização florestal. Piracicaba: IPEF, 2000. p. 105-133.

Bertacchi, M. I. F. et al. Establishment of tree seedlings in the understory of restoration plantations: natural regeneration and enrichment plantings. Restoration Ecology, v. 24, n. 1, p. 100-108, 2016. DOI: $10.1111 /$ rec. 12290.

Campoe, O. C. et al. Atlantic forest tree species responses to silvicultural practices in a degraded pasture restoration plantation: from leaf physiology to survival and initial growth. Forest Ecology and Management, v. 313, p. 233-242, 2014. DOI: 10.1016/ j.foreco.2013.11.016.

Cruz, C. A. F. et al. Resposta de Mudas deSenna macranthera (Dc. Ex coollad.) H. S. Irmin \& Barnaby (fedegoso) Cultivadas em Latossolo Vermelho-Amarelo Distrófico a Macronutrientes. Revista Árvore, v. 34, n. 1, p. 13-24, 2010. DOI: 10.1590/S0100-67622010000100002.

Davide, A. C. et al. Fatores que afetam a qualidade de mudas destinadas aos projetos de restauração de ecossistemas florestais. In: Davide, A. C. \& Botelho, S. A. Fundamentos e métodos de restauração de ecossistemas florestais: 25 anos experiência em mata ciliares. Lavras: Ed. da UFLA, 2015. p. 181-274.
Dickson, A. et al. Quality appraisal of while spruce and white pine seedling stock in nurseries. Forestry Chronicle, v. 36, p. 11-13, 1960. DOI: $10.5558 / \mathrm{tfc} 36010-1$.

Dutra, A. F. et al. Substrate and irrigation scheme on the growth of Parapiptadenia rigida (angico-vermelho) seedlings. Ciência Rural, v. 46, n. 6, p. 1007-1013, 2016. DOI: 10.1590/0103-8478cr20141732.

Ferez, A. P. C. et al. Silvicultural opportunities for increasing carbon stock in restoration of Atlantic forests in Brazil. Forest Ecology and Management, v. 350, p. 40-45, 2015.

Ferrari, M. et al. Influência de fertilizante de liberação controlada em mudas de Tabernaemontana catharinensis. Pesquisa Florestal Brasileira, v. 36, n. 88, p. 543-547, 2016. DOI: 10.4336/2016. pfb.36.88.968.

Ferreira, D. F. Sisvar: a guide for its bootstrap procedures in multiple comparisons. Ciência e Agrotecnologia, v. 38, n. 2, p. 179-182, 2014. DOI: $10.1590 / \mathrm{S} 1413-70542014000200001$.

Fonseca, E. P. et al. Padrão de qualidade de mudas de Trema micrantha (L.) Blume, produzidas sob diferentes períodos de sombreamento. Revista Árvore, v. 26, n. 4, p. 515-523, 2002. DOI: 10.1590/S0100-67622002000400015.

Freitas, T. A. S. et al. Desempenho radicular de mudas de eucalipto produzidas em diferentes recipientes e substratos. Revista Árvore, v. 29, n. 6, p. 853-861, 2005. DOI: 10.1590/S0100-67622005000600003

Gasparin, E. et al. Influência do substrato e do volume de recipiente na qualidade das mudas de Cabralea canjerana (Vell.) Mart. em viveiro e no campo. Ciência Florestal, v. 24, n. 3, p. 553-563, 2014. DOI: $10.1590 / 1980-509820142403004$.

Gomes, J. M. \& Paiva, H. N. Viveiros florestais: propagação sexuada. Viçosa, MG: Ed. da UFV, 2011. 116 p. (Série didática).

Gonçalves, E. O. et al. Nutrição de mudas de angico-vermelho (Anadenanthera macrocarpa (Benth.) Brenan) submetidas a doses de N, P, K, Ca e Mg. Revista Árvore, v. 36, n. 2, p. 219-228, 2012. DOI: $10.1590 / \mathrm{S} 0100-67622012000200003$.

Kämpf, A. N. Produção comercial de plantas ornamentais. 2. ed. Guaíba: Agrolivros, 2005. 256 p.

Knapik, J. G. \& Angelo, A. C. Pó de basalto e esterco eqüino na produção de mudas de Prunus sellowii Koehne (Rosaceae). Floresta, v. 37 , n. 3 , p. $427-436$, 2007. DOI: 10.5380/rf.v37i3.9939.

Melo, L. A. et al. Crescimento de mudas de Eucalyptus grandis e Eremanthus erythropappus sob diferentes formulações de substrato. Floresta e Ambiente, v. 21, p. 234-242, 2014. DOI: 10.4322/ floram.2014.028.

Mezzomo, J. C. et al. Does the quality of Luehea divaricata seedlings in the nursery correspond to their behavior in the field? African Journal of Agricultural Research, v. 12, n. 8, p. 658-663, 2017. DOI: 10.5897/AJAR2016.11881.

Regan, R. P. Evaluating alternative growing media components. In: Wilkinson, K. M. et al. (Ed.). National proceedings: Forest and Conservation Nursery Associations: 2013. Fort Collins: U.S. Department of Agriculture, Forest Service, Rocky Mountain Research Station, 2014. p. 50-53. 
Rorato, D. G. et al. Tolerance and resilience of forest species to frost in restoration planting in Southern Brazil. Restoration Ecology, v. 25, n. 5, 2017. DOI: 10.1111/rec. 12596 .

Saidelles, F. L. F. et al. Casca de arroz carbonizada como substrato para produção de mudas de tamboril-da-mata e garapeira. Semina, v. 30, p. 1173-1186, 2009. DOI: 10.5433/1679-0359.2009v30n4Sup1p1173.
Soares, F. C. et al. Consumo de água pela cultura do lírio, cultivado em substratos alternativos em condições de ambiente protegido. Ciência Rural, v. 42, n. 6, p. 1001-1006, 2012. DOI: 10.1590/S0103-84782012000600008. 